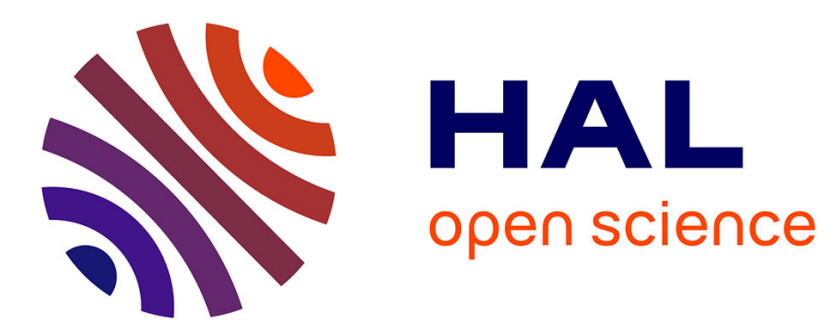

\title{
Robust Stability Analysis of a Class of Smith Predictor-based Congestion Control Algorithms for Computer Networks
}

\author{
Luca de Cicco, Saverio Mascolo, Silviu-Iulian Niculescu
}

\section{To cite this version:}

Luca de Cicco, Saverio Mascolo, Silviu-Iulian Niculescu. Robust Stability Analysis of a Class of Smith Predictor-based Congestion Control Algorithms for Computer Networks. 8th IFAC Workshop on Time-Delay Systems, Sep 2009, Sinaia, Romania. pp.201-206, 10.3182/20090901-3-RO-4009.00032 . hal-02291626

\section{HAL Id: hal-02291626 \\ https://hal.science/hal-02291626}

Submitted on 19 Sep 2019

HAL is a multi-disciplinary open access archive for the deposit and dissemination of scientific research documents, whether they are published or not. The documents may come from teaching and research institutions in France or abroad, or from public or private research centers.
L'archive ouverte pluridisciplinaire HAL, est destinée au dépôt et à la diffusion de documents scientifiques de niveau recherche, publiés ou non, émanant des établissements d'enseignement et de recherche français ou étrangers, des laboratoires publics ou privés. 


\title{
Robust Stability Analysis of a Class of Smith Predictor-based Congestion Control Algorithms for Computer Networks
}

\author{
Luca De Cicco* Saverio Mascolo* Silviu-Iulian Niculescu** \\ * Politecnico di Bari, Dipartimento di Elettrotecnica ed Elettronica, \\ Via Orabona, 4, Bari, Italy (e-mail: \{ldecicco, mascolo\}@poliba.it). \\ ** Supelec, Laboratoire des Signaux et Systemes, 3 rue Joliot-Curie \\ 91192 Gif-sur-Yvette cedex, France (e-mail: \\ Silviu.Niculescu@lss.supelec.fr)
}

\begin{abstract}
Congestion control is a fundamental building block in packet switching networks such as the Internet due to the sharing of communication resources. It has been shown that the plant dynamics is essentially made of an integrator plus time delay and that a proportional controller plus a Smith predictor is a simple and effective controller. It has been also shown that the today running TCP congestion control can be modelled using a Smith predictor plus a proportional controller. Due to the importance of this control structure in the field of data network congestion control, we analyze the robust stability of the closed loop system in the face of delay uncertainties that in data networks are present due to queuing. In particular, by applying a geometric approach, we derive a bound on the proportional controller gain which is necessary and sufficient to guarantee stability given a bound on the delay uncertainty.
\end{abstract}

Keywords: Time-delay systems, Robust stability, congestion control, Smith predictor

\section{INTRODUCTION}

Time delays are often present in feedback control systems due to reasons such as the transport of material or information. From the control theoretic point of view it is well-known that an increase of the time delay may lead to instability of the closed loop system and to performance degradation as well.

The Smith principle is a classic approach which is often employed to design controllers for time delay systems (Smith (1959)). It is known that, by assuming exact knowledge of both the plant model and time delay, controllers designed using a Smith predictor are very effective in counteracting the effect of time delays. Robustness of the Smith predictor with respect to uncertainties in the knowledge of the time delay has been extensively studied since 1980 (Palmor (1980), Yamanaka and Shimemura (1987)).

The Internet represents a relevant example of time delay system due to the presence of delays that are caused by the propagation of the information, which is sent in form of data packets, from a source to a destination through a series of communication links and router queues.

A cornerstone component of the Internet protocol stack is the end-to-end congestion control which has been implemented in the TCP by Jacobson (1988) in order to avoid congestion and preserve network stability. Several fluid models have been proposed for the TCP congestion control algorithm in order to analytically study the stability of the network under different scenarios. See Mascolo (1999); Hollot et al. (2002); Srikant (2004) and references therein.
In Mascolo (1999) a simple model of the plant made of an integrator (modelling the bottleneck queue) plus two time delays (modelling forward and backward delays), has been proposed along with a Smith predictor plus a proportional controller. The paper also shows that the Smith predictor controller with a proportional gain models the congestion control law which is employed in the today running TCP congestion control algorithm. Moreover, the model presented in Mascolo (1999) has been employed in Grieco and Mascolo (2004) to design and implement a ratebased congestion control algorithm which has been found to produce flows that are TCP-friendly.

A similar plant model is employed in Quet et al. (2002) to design a rate-based congestion control algorithm implemented at the router that is robust to uncertain timedelays by employing the $\mathcal{H}^{\infty}$ technique. The controller parameters are quite complex to derive and, at the best of authors knowledge, no real implementations are currently available to assess the effectiveness of the proposed solution.

Measurement of the plant time delay to be used in the Smith predictor can be affected by uncertainties due to the fact that the time delay is made of a constant propagation delay plus time-varying queueing delays. To the purpose, the standard TCP (Postel (1981)) estimates the Round Trip Time (RTT) through time-stamping in order to set the retransmission timeout (RTO) which is needed for detecting heavy congestion episodes in the network. The RTT is defined as the time that elapses from when a segment is sent until the corresponding acknowledgement segment is received by the sender. In 
the standard TCP implementation, the RTT is measured each RTT seconds, whereas no measurements are taken on retransmitted segments due to the Karn's algorithm in order to avoid spurious timeouts (see Karn and Partridge (1987)). For these reasons the standard TCP does not provide an accurate measure of RTT. In order to overcome this issue an optional scheme has been proposed and standardized in Jacobson et al. (1992) which makes use of timestamps in an optional field of the TCP header. However, even if the timestamp option is employed by both peers of the communication, the granularity chosen for TCP timestamps is implementation-dependent. In a recent work Veal et al. (2005) carry out an extensive measurement campaign on RTTs. Authors used 500 servers and found that $76 \%$ of the servers had timestamping option enabled, and out of these servers $37 \%$ used a $100 \mathrm{~ms}$ granularity, $55 \%$ a $10 \mathrm{~ms}$ granularity and only $7 \%$ of them had a granularity of $1 \mathrm{~ms}$.

A preliminary study on robust stability of a proportional Smith predictor used for congestion control in data networks has been carried out by using the Nyquist criterion in Mascolo (2003). It revealed that in order to guarantee asymptotic stability it is sufficient that $\Delta<1 / k$ where $\Delta$ represents the delay uncertainty and $k$ is the gain of the proportional controller.

The goal of this paper is to provide a characterization of the robust stability of system introduced in Mascolo (1999) by applying the geometric approach which has been developed in $\mathrm{Gu}$ et al. (2005).

The rest of the paper is organized as follows: in Section 2 we briefly review the model of the closed loop congestion control in a generic packet switching network presented in Mascolo (1999); in Section 3 we apply the geometrical approach developed in Morarescu et al. (2006) in order to find the stability crossing curves of the system; in Section 4 we present the robust stability analysis; in Section 5 some simulations are presented to support the theoretical results obtained; finally Section 6 concludes the paper.

\section{CONGESTION CONTROL MODEL}

A network connection is basically made by a set of communication links and store-and-forward nodes (routers) where packets are enqueued before being routed to the destination. Congestion can arise when packets arrive at a rate $r(t)$ which is above the capacity of the output link so that the router queue builds up until it is full and it starts to drop packets.

In Mascolo (1999) a model of the Internet flow and congestion control as a time delay system is provided and in Mascolo (2006) it is shown that different variants of TCP congestion control algorithms can be modelled in a unified framework by proper input shaping of the proportional Smith predictor controller.

In particular, the model consists of a feedback loop in which two time delays are present as it is shown in Figure 1: $\tau_{f w}$ models the propagation time of a packet from source to the bottleneck queue and $\tau_{f b}$ models the propagation time from the bottleneck to the destination and then back to the sender. The round trip time of the connection is $\tau=\tau_{f w}+\tau_{f b}$.

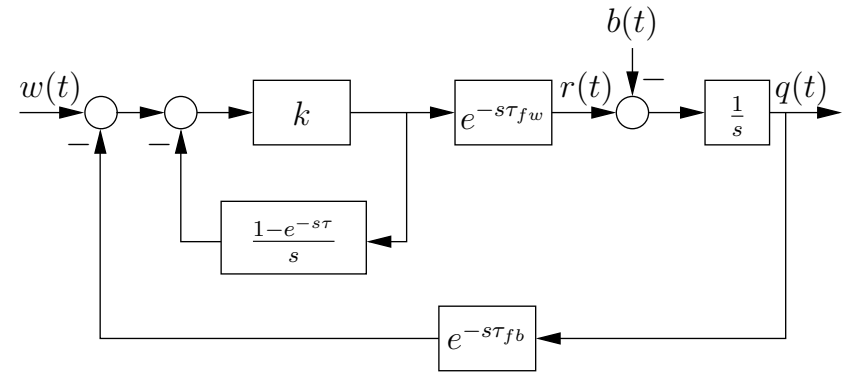

Figure 1. Functional block of the congestion control model

The simple integrator $1 / s$ models the bottleneck queue that is filled (or drained) by the rate mismatch $r(t)-b(t)$, where $b(t)$ is the bottleneck available bandwidth.

The controller is a proportional Smith predictor with gain $k$ that computes the rate $r(t)$ to match the available bandwidth $b(t)$ and to produce a stable output. The reason for using a simple proportional controller is that in this way the closed-loop dynamics can be made that of a first-order system with time constant $1 / k$ delayed by $\tau_{f w}$. Thus, the step response of the system can be made faster by increasing the proportional gain $k$ providing an always stable system without oscillations or overshoots. This choice provides a controller in which only one design parameter, i.e. the gain $k$, has to be tuned having a direct influence on the dynamics of the output. Finally, the input signal $w(t)$ models the congestion window (cwnd) or the advertised window, that is used by the congestion control algorithm to bound in-flight packets (Mascolo (1999)).

Model mismatches are known to affect the closed loop dynamics when a Smith predictor controller is employed. In this case, it is worth noting that the only source of mismatch between the model and the actual plant is the entity of the delay (see Section 1) whereas the model of the bottleneck queue is an integrator and does not add any uncertainty. In the next sections we will give simple tuning rules for the design parameter $k$ in order to retain asymptotic stability when the measure of time delay $\tau$ is uncertain.

Finally, we remark that a Smith predictor controller is recommended when designing a congestion control algorithm for data networks, since using PID controllers would provide an unacceptable sluggish system due to large delays involved in communication networks (see Astrom and Hagglund (1995) and Mascolo (1999)).

\section{STABILITY CROSSING CURVES IN THE PARAMETERS SPACE}

\subsection{Review of the geometrical approach}

We start by briefly reviewing the geometrical approach developed in Morarescu et al. (2006) which we will employ to analyze the robust stability of the considered system. The reader is advised to refer to $\mathrm{Gu}$ et al. (2005) for a complete description of the method. We denote with $a\left(s ; \tau_{1}, \tau_{2}\right)$ the characteristic function of the closed-loop system where $\tau_{1}$ represents the nominal delay used in the Smith predictor and $\tau_{2}=\tau_{1}+\Delta$ represents the actual plant delay affected by a bounded mismatch $\Delta$. It is easy 


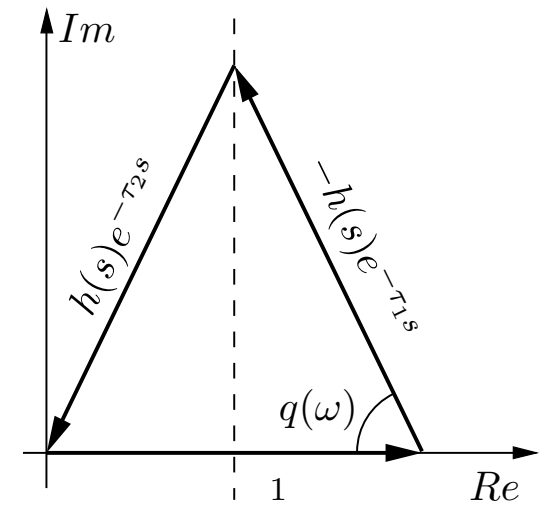

Figure 2. Triangle formed by the three vectors when the characteristic equation holds

to show that the characteristic function in this case is given by:

$$
a\left(s ; \tau_{1}, \tau_{2}\right)=1-h(s) e^{-\tau_{1} s}+h(s) e^{-\tau_{2} s}
$$

where $h(s)$ is the transfer function of the closed loop system when no delays are present in the loop:

$$
h(s)=\frac{C(s) G_{0}(s)}{1+C(s) G_{0}(s)}
$$

with $G_{0}(s)$ being the delay free plant and with $C(s)$ being the controller transfer function.

In order to analyze the stability of the system we look for the solutions of the characteristic equation:

$$
a\left(j \omega ; \tau_{1}, \tau_{2}\right)=0
$$

In this way we are able to find all the conditions under which the system has at least one pole on the imaginary axis. The geometrical approach relies on the observation that the three terms of the characteristic function (1) can be seen as vectors in the complex plane. Therefore, the equality $a\left(s ; \tau_{1}, \tau_{2}\right)=0$ can be represented in the complex plane via an isosceles triangle as it is shown in Figure 2. Thus, equation (2) is equivalent to the following three conditions:

(1) The triangular inequality must hold for the triangle shown in Figure 2, which implies that:

$$
|h(j \omega)| \geq \frac{1}{2}
$$

(2) Equation (2) must satisfy the phase rule;

(3) The sum of the internal angles of the isosceles triangle must be equal to $\pi$;

The solution of (3), which does not depend on time delays $\tau_{1}$ or $\tau_{2}$, forms the frequency crossing set $\Omega$ which is the union of a finite number $N$ of intervals of finite length $\Omega_{1}, \Omega_{2}, \ldots, \Omega_{N}$. For any $\omega>0$ which belongs to the frequency crossing set there exists at least a pair $\left(\tau_{1}, \tau_{2}\right)$ in the parameters space such that the system has at least one imaginary pole. The conditions 2 and 3 imply that for all $\omega \in \Omega$ all the couples $\left(\tau_{1}, \tau_{2}\right) \in \mathbb{R}_{+}^{2}$ satisfying $a\left(j \omega ; \tau_{1}, \tau_{2}\right)=0$ can be found using the following equations:

$$
\tau_{1}^{u \pm}=\frac{\angle h(j \omega)+2 u \pi \pm q(\omega)}{\omega}
$$

$$
\tau_{2}^{v \pm}=\frac{\angle h(j \omega)+(2 v-1) \pi \mp q(\omega)}{\omega}
$$

where $u$ and $v$ are integers such that the corresponding $\tau_{1}^{u \pm}, \tau_{2}^{v \pm}$ are non negative and $q(\omega)$ represents the internal angle of the isosceles triangle:

$$
q(\omega)=\arccos \left(\frac{1}{2|h(j \omega)|}\right)
$$

In order to understand the meaning of equations (4) and (5) let us fix $u=\bar{u}, v=\bar{v}$ and consider the set $\Omega_{i} \subseteq \Omega$ : if $\omega$ varies in $\Omega_{i}$ and we evaluate (4) and (5) for both positive and negative signs we obtain two curves in the parameter space $\left(\tau_{1}, \tau_{2}\right)$ which we denote $\mathcal{T}_{\bar{u}}^{i}, \bar{v}$ and $\mathcal{T}_{\bar{u}}^{i-}$ respectively. It is worth noting that the curves $\mathcal{T}_{u, v}^{i}=\mathcal{T}_{u, v}^{i-} \cup \mathcal{T}_{u, v}^{i+}$ can be either open curves or closed curves depending on the set $\Omega_{i}$ we are considering. In particular, it is easy to show that if the left end of $\Omega_{i}$ is 0 then the associated curve is an open curve with both ends approaching $\infty$ when $\omega \rightarrow 0$. On the other hand, if the left end of $\Omega_{i}$ is not 0 then $\mathcal{T}_{\bar{u}}^{i} \bar{v}$ is a closed curve ( $\mathrm{Gu}$ et al. (2005)).

We define the stability crossing curves $\mathcal{T}$ in the $\tau_{1}, \tau_{2}$ plane as the union of all the curves $\mathcal{T}_{u, v}^{i}$ when $i \in\{1, \ldots, N\}$, and $u$ and $v$ vary in the set of integers.

Finally, it is important to point out that when a stability crossing curve is crossed in the $\tau_{1}, \tau_{2}$ plane, two poles cross the imaginary axis on the complex plane ( $\mathrm{Gu}$ et al. (2005)).

\subsection{Stability crossing curves of the computer network congestion control model}

In order to characterize the impact of the delay uncertainty on the stability of the considered feedback system we apply the geometric approach we have reviewed in Section 3.1. It is worth to notice that the delay-free model of the plant is $G_{0}(s)=1 / s$ and the controller transfer function is $C(s)=k$.

We suppose that the system described in Section 2 is affected by a delay uncertainty $\Delta$ which is bounded by $\delta>0$, i.e. $|\Delta|<\delta$. By considering the delay uncertainty, the characteristic equation of the system can be rewritten as follows:

$$
1+\frac{k}{s}-\frac{k}{s} e^{-\tau_{1} \cdot s}\left(1-e^{-\Delta \cdot s}\right)=0
$$

where $\tau_{1}$ represents the nominal round trip time (RTT) of the considered connection, which is used in the Smith predictor, and $\tau_{2}=\tau_{1}+\Delta$ is the actual plant time delay.

By multiplying by $s /(s+k)$ both sides of (6) we obtain:

$$
1-\frac{k}{s+k} e^{-\tau_{1} s}+\frac{k}{s+k} e^{-\left(\Delta+\tau_{1}\right) s}=0
$$

so that by considering $h(s)=k /(s+k)(7)$ is in the form of (1). We are interested in characterizing the stability of the system when $\tau_{1}, \tau_{2}$ and $k$ vary in $\mathbb{R}_{+}$.

By making the change of variable $z=s / k$ we obtain:

$$
1-\frac{1}{z+1} e^{-h_{1} z}+\frac{1}{z+1} e^{-h_{2} z}=0
$$

where $h_{1}=k \tau_{1}$ and $h_{2}=k \tau_{2}$, which reduces the free parameters to two. It is worth to notice that the 


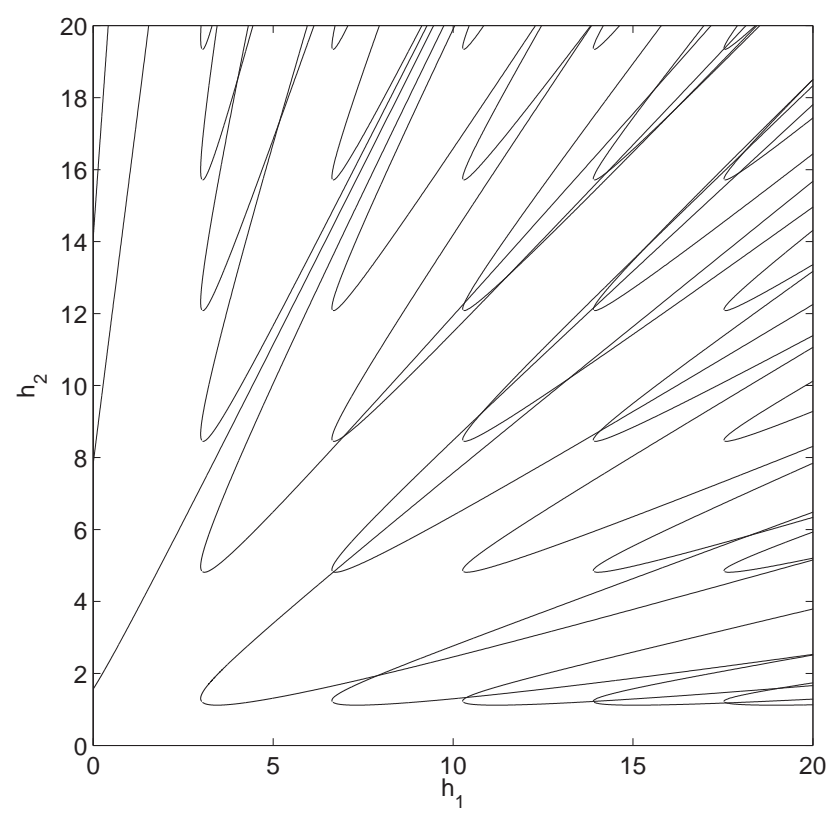

Figure 3. Stability crossing curves for the considered system

transformation from (7) to (8) simply involves a scaling of the closed-loop eigenvalues by $1 / k$, thus indicating a natural trade-off between gain and delay since when $k$ increases the closed loop poles approach to the imaginary axis (Niculescu and Michiels (2004)).

We are now ready to study the stability of the original system in the $h_{1}, h_{2}$ plane regardless the value of the proportional gain $k$.

First of all, by applying (3) we find that the crossing set is made by the single interval $\Omega=[0, \sqrt{3}]$ which means that the stability crossing curves in the $h_{1}, h_{2}$ plane are open curves which extend to infinity when $\omega \rightarrow 0$. By using (4) and (5) the stability crossing curves of the considered system result the following:

$$
\begin{array}{r}
h_{1}^{u \pm}(\omega)=\frac{-\arctan \omega+2 u \pi \pm \arccos \left(\frac{\sqrt{1+\omega^{2}}}{2}\right)}{\omega} \\
h_{2}^{v \pm}(\omega)=\frac{-\arctan \omega+(2 v-1) \pi \mp \arccos \left(\frac{\sqrt{1+\omega^{2}}}{2}\right)}{\omega}
\end{array}
$$

Figure 3 shows the stability crossing curves of the considered system. We start by considering the $h_{2}$ axis $\left(h_{1}=0\right)$, which means that we are employing a simple proportional controller without the Smith predictor. By starting from the origin and increasing the value of $h_{2}$ the first curve is crossed at $h_{2}=\pi / 2$ which means that the system becomes unstable for $h_{2}>\pi / 2$ as expected. On the other hand, the axis $h_{1}$ represents the system in which no delay affects the plant, but the Smith predictor is in the controller. Figure 3 shows that the system is stable for all the delays in the Smith predictor.

Finally, points on the positive bisector represent the case of perfect matching of nominal delay $\tau_{1}$ with the actual delay $\tau_{2}$. Indeed, if we move on this line no curves will be crossed since the Smith predictor in this case provides a stable system regardless the value of the proportional gain $k$.

\section{ROBUST STABILITY ANALYSIS}

In this Section we will develop an analysis of the robust stability of the considered system by using the stability crossing curves we have shown in the previous Section. We already know that the considered system is always asymptotically stable for any delay $\tau_{1}$ and any proportional gain $k$ as far as the delay uncertainty is zero thanks to the perfect compensation of the time delay $\tau_{1}$ provided by the Smith predictor. In the $h_{1}, h_{2}$ plane this condition means that the system is asymptotically stable on all the positive bisector.

In order to characterize the robustness of the system in the face of delay uncertainties we compute the maximum delay mismatch which still preserves stability. Thus, the problem here is to look for the maximum deviation $\delta$ with respect to a generic point $\left(\tau_{1}^{*}, \tau_{1}^{*}\right)$ with $\tau_{1}^{*} \geq 0$ which lies on the positive bisector such that the system is stable for any $\left(\tau_{1}, \tau_{2}\right)$ which satisfies:

$$
\left|\tau_{2}-\tau_{1}^{*}\right|<\delta
$$

We remark that solving the maximum admissible delay uncertainty problem is equivalent to find the minimum distance between the stability crossing curves and a generic point on the positive bisector of the $h_{1}, h_{2}$ plane.

Thus for any $\tau_{1}^{*}>0$ we have to solve:

$$
\delta\left(\tau_{1}^{*}\right)=\min _{u, v} \min _{\tau_{2}^{v \pm} \in \mathcal{T}}\left|\tau_{2}^{v \pm}-\tau_{1}^{*}\right|
$$

so that the maximum delay to retain stability is:

$$
\delta=\min _{\tau_{1}^{*} \in \mathbb{R}_{+}} \delta\left(\tau_{1}^{*}\right)
$$

Proposition 1. A necessary and sufficient condition for the asymptotic stability of the system regardless the value of the nominal delay $\tau_{1}$ is:

$$
|\Delta|<\frac{\alpha}{k}
$$

where $\Delta$ is the delay uncertainty, $\alpha \cong 1.4775$ and $k$ is the proportional gain of the controller.

\section{Proof.}

We start by considering the stability crossing curves in the parameters space $h_{1}, h_{2}$. In order to find the minimum distance between the stability crossing curves and a generic point of positive bisector of the $h_{1}, h_{2}$ plane we evaluate the tangent to the crossing curves with direction parallel to the positive bisector:

$$
\frac{d h_{2}}{d h_{1}}=1 \Leftrightarrow \frac{d h_{2}}{d \omega} \frac{d \omega}{d h_{1}}=1 \Leftrightarrow \frac{d h_{1}}{d \omega}=\frac{d h_{2}}{d \omega}
$$

To the purpose we look for a subset $\overline{\mathcal{T}}$ of the stability crossing curves $\mathcal{T}$ that are the "closest" curves to the positive bisector. By considering a generic curve $\mathcal{T}_{u, v}$ and by applying (9) and (10) it turns out that for all $u$ and $v$ and for all $\omega \in \Omega$ it holds $h_{2}^{v+}-h_{1}^{u+}<h_{2}^{v-}-h_{1}^{u-}$ so that it is sufficient to consider only the curves $\mathcal{T}_{u, v}^{+}$ in the region $h_{2}>h_{1}$ and the curves $\mathcal{T}_{u, v}^{-}$in the region 
$h_{2}<h_{1}$, since they will be the closest ones to the positive bisector. Thus, we can refer without loss of generality to the generic curves of $\mathcal{T}$ as $\mathcal{T}_{u, u+i}$ for all $i$ and $u$ in the integers. Straightforward computations on (9) and (10) give:

$$
h_{2}^{u+i}-h_{1}^{u}>h_{2}^{u+i-1}-h_{1}^{u}
$$

which means that when $i$ decreases the curves $\mathcal{T}_{u, u+i}$ will move downwards in the $h_{1}, h_{2}$ plane. Figure 4 shows the values of $u$ and $v$ for the curves $\mathcal{T}_{u, u}$ and $\mathcal{T}_{u, u+1}$. It is then easy to show that if we set $v=u$ we obtain the closest curves to the positive bisector in the region $h_{2}<h_{1}$ whereas the curves with $v=u+1$ are those which are closest to the positive bisector in the region $h_{2}>h_{1}$. In conclusion we can restrict our search to the set :

$$
\overline{\mathcal{T}}=\mathcal{T}_{u, u}^{-} \cup \mathcal{T}_{u, u+1}^{+}
$$

for all $u$ in the integers. Let us consider the region $h_{2}>h_{1}$ i.e. we consider the subset $\mathcal{T}_{u, u+1}^{+}$. By considering (14) after straightforward computations we get the following equation:

$$
\begin{gathered}
\arccos \left(\frac{\sqrt{\omega^{2}+1}}{2}\right)+\frac{\omega^{2}}{\sqrt{\omega^{2}+1} \sqrt{3-\omega^{2}}}+ \\
+\pi\left(v-u-\frac{1}{2}\right)=0
\end{gathered}
$$

with $\omega \in \Omega$. By letting $v=u+1$ the equation (15) has the unique solution $\bar{\omega}=1.3483 \mathrm{rad} / \mathrm{s}$ in $\Omega$ which is independent of $u$. If we substitute this value in (9) and (10) we obtain:

$$
\begin{aligned}
& h_{1}(\bar{\omega})=h_{1}=4.6601 u-0.2654 \\
& h_{2}(\bar{\omega})=h_{2}=4.6601 v-3.4480
\end{aligned}
$$

Thus, all the points belonging to the the curves $\mathcal{T}_{u, u+1}^{+}$ having a tangent which is parallel to the positive bisector, lie on the line:

$$
h_{2}=h_{1}+1.4775
$$

For this reason we can conclude that the maximum uncertainty, in the $h_{1}, h_{2}$ coordinates is 1.4775 . The proof is completed by recalling that $h_{1}=k \tau_{1}$ and $h_{2}=k \tau_{2}$ and that $\tau_{2}=\tau_{1}+\Delta$. Thus, we finally obtain:

$$
h_{2}-h_{1}<1.4775 \Rightarrow k \Delta<1.4775 \Rightarrow \Delta<\frac{1.4775}{k}
$$

It is worth to notice that the same procedure can be followed in the case $v=u$ which leads to the inequality:

$$
h_{1}-h_{2}<1.4775 \Rightarrow-k \Delta>1.4775 \Rightarrow \Delta>-\frac{1.4775}{k}
$$

Thus, by considering both (17) and (18) we obtain (13). In order to prove the necessity of the condition (13) let us consider the curves $\mathcal{T}_{u, u+1}$. The points of the curve $\mathcal{T}_{u, u+1}$ that correspond to the frequency $\omega=1.3483 \mathrm{rad} / \mathrm{s}$ lie on the line described by (16) so that the maximum delay uncertainty admissible for those points is exactly $\alpha / k$. If we select a larger value for $\delta$ the system will become unstable at least on those points. This concludes the proof. Remark 2. The fact that the maximum uncertainty allowed does not depend on the nominal delay $\tau_{1}$ is a nice

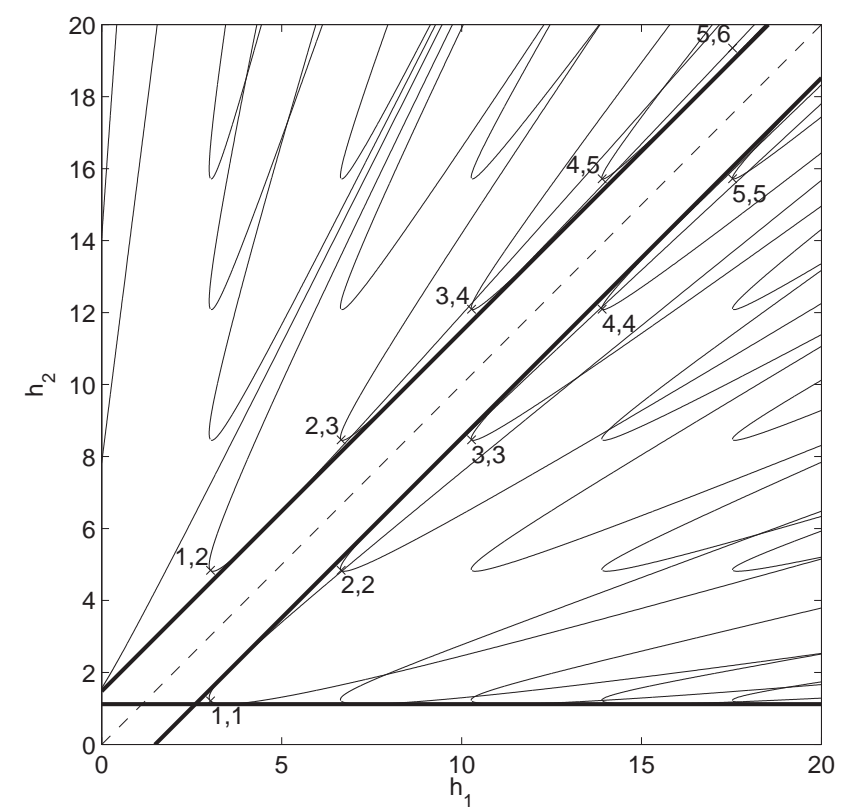

Figure 4. Stability regions for the considered system

feature of the Smith predictor based controller. This makes the controller effective even with large delays.

Remark 3. The condition (13) expresses a trade-off between the maximum delay mismatch $\delta$ and the proportional gain that can be used to tune the controller gain $k$.

Remark 4. This result improves the robust stability condition $|\Delta|<1 / k$ found in Mascolo (2003).

Proposition 5. The system is stable, regardless the value of $\tau_{1}$, if the delay uncertainty $\Delta$ satisfies the following inequality:

$$
-\tau_{1}<\Delta<-\tau_{1}+\frac{\beta}{k}
$$

with $\beta=1.1188$.

\section{Proof.}

The proof follows the same arguments of Proposition 1, therefore it is omitted.

Remark 6. The condition (19) implicitly requires the delay uncertainty $\Delta$ to be negative, i.e. the nominal delay $\tau_{1}$ should be always below the actual delay of the plant $\tau_{2}$. Thus, condition (19) has no particular meaning for the characterization of controller robustness, since the sign of the uncertainty is not known a priori.

\section{SIMULATION RESULTS}

In this Section we report simulation results obtained by using a SIMULINK model that implements the system depicted in Figure 1. The bottleneck available bandwidth has been set to vary as a step function starting at time $t=1 \mathrm{sec}$ and having a final value of $b=100$ packets $/ \mathrm{sec}$. The queue set-point is a step function starting at $t=0 \mathrm{sec}$ with a final value of $w=150$ packets. The gain of the controller has been set to $k=4 \mathrm{sec}^{-1}$ corresponding to a maximum delay uncertainty of $\delta \cong 0.37 \mathrm{sec}$. The nominal $R T T$ of the connection is $1 \mathrm{sec}$. Figure 5 reports the queue 


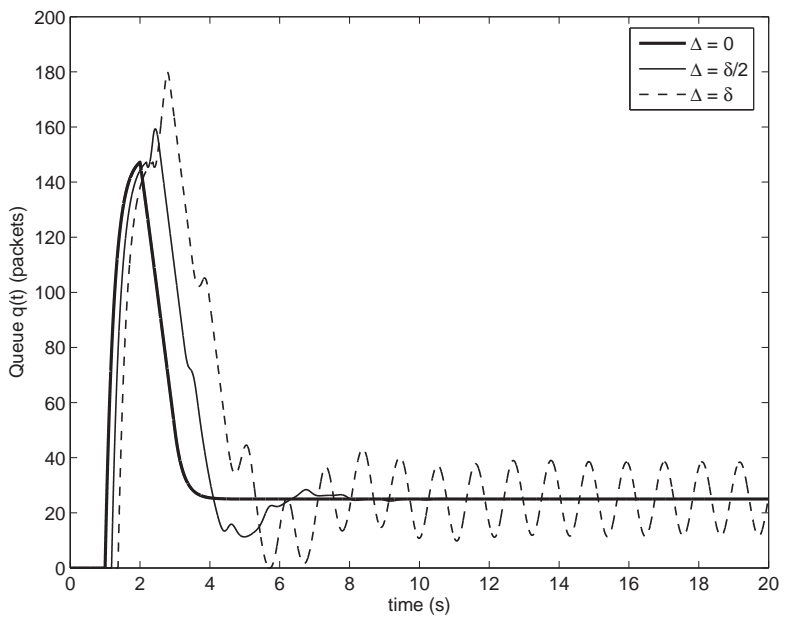

(a)

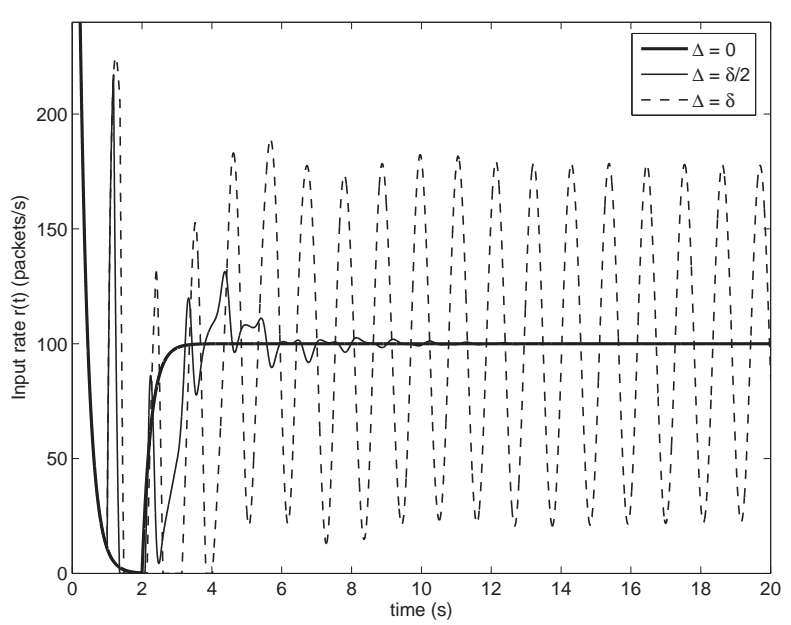

(b)

Figure 5. Queue length (a) and input rate evolution (b) when $\tau_{1}=1 \mathrm{sec}, k=4 \mathrm{sec}^{-1}, b(t)=100 \mathrm{packets} / \mathrm{sec}$, $w=150$ packets

evolution $q(t)$ and the input rate $r(t)$ when the delay uncertainty is either zero, $\delta / 2 \cong 0.185 \mathrm{sec}$ or $\delta \cong 0.37 \mathrm{sec}$.

The figure shows, as expected, that the performance of the closed loop response degrades when the delay uncertainty increases. In particular, oscillations are present when the delay uncertainty is $\delta / 2$ still providing an acceptable response, whereas when the delay uncertainty increases to the maximum allowed value persistent oscillations occur.

\section{CONCLUSIONS}

In this paper we have analyzed the robust stability of a very important class of congestion control algorithms when delay uncertainties are present. We have shown how the geometrical approach developed in Morarescu et al. (2006) can be easily applied in order to find stability bounds on the parameter of the controller. Moreover, we found a simple necessary and sufficient condition on the gain of the proportional controller $k$ in order to retain asymptotic stability regardless the value of the nominal delay $\tau_{1}$. Such a result suggests that congestion control algorithms that employ controllers made by a Smith predictor plus a proportional gain can be easily tuned in order to be robust to a bounded delay uncertainty.

\section{REFERENCES}

Astrom, K. and Hagglund, T. (1995). PID Controllers: Theory, Design, and Tuning. Instrument Society of America, 80-81.

Grieco, L.A. and Mascolo, S. (2004). Adaptive rate control for streaming flows over the internet. ACM Multimedia Systems Journal, 9(6), 517-532.

Gu, K., Niculescu, S., and Chen, J. (2005). On stability crossing curves for general systems with two delays. Journal of mathematical analysis and applications, 311(1), 231-253.

Hollot, C., Misra, V., and Towsley, D. (2002). Analysis and design of controllers for AQM routers supporting TCPflows. Automatic Control, IEEE Transactions on, 47(6), 945-959.

Jacobson, V. (1988). Congestion avoidance and control. ACM SIGCOMM '98.

Jacobson, V., Braden, R., and Borman, D. (1992). TCP Extensions for High Performance. RFC 1323, standard.

Karn, P. and Partridge, C. (1987). Estimating round-trip times in reliable transport protocols. In Proc. $A C M$ SIGCOMM '87, volume 17.

Mascolo, S. (1999). Congestion control in high-speed communication networks using the Smith principle. Special Issue on "Control methods for communication networks" Automatica, 35(12), 1921-1935.

Mascolo, S. (2003). Modeling the Internet congestion control as a time delay system: a robust stability analysis. In Proc. the IFAC workshop on time-delay systems.

Mascolo, S. (2006). Modeling the Internet congestion control using a Smith controller with input shaping. Control engineering practice, 14(4), 425-435.

Morarescu, C., Niculescu, S., and Gu, K. (2006). On the geometry of stability regions of Smith predictors subject to delay uncertainty. IMA Journal of Mathematical Control and Information.

Niculescu, S. and Michiels, W. (2004). Stabilizing a chain of integrators using multiple delays. Automatic Control, IEEE Transactions on, 49(5), 802-807.

Palmor, Z. (1980). Stability properties of Smith deadtime compensator controllers. International Journal of Control, 32(6), 937-949.

Postel, J. (1981). Transmission Control Protocol. RFC 793, Standard.

Quet, P., Ataşlar, B., Iftar, A., Özbay, H., Kalyanaraman, S., and Kang, T. (2002). Rate-based flow controllers for communication networks in the presence of uncertain time-varying multiple time-delays. Automatica, 38(6), 917-928.

Smith, O. (1959). A controller to overcome dead time. ISA Journal, 6(2), 28-33.

Srikant, R. (2004). The Mathematics of Internet Congestion Control. Birkhäuser.

Veal, B., Li, K., and Lowenthal, D. (2005). New Methods for Passive Estimation of TCP Round-Trip Times. In Proc. Passive And Active Network Measurement (PAM) '05. Springer.

Yamanaka, K. and Shimemura, E. (1987). Effects of mismatched Smith controller on stability in systems with time-delay. Automatica, 23(6), 787-791. 